\title{
On the equivalence of linear complementarity problems*
}

\author{
B. De Schutter, W.P.M.H. Heemels, and A. Bemporad
}

If you want to cite this report, please use the following reference instead:

B. De Schutter, W.P.M.H. Heemels, and A. Bemporad, "On the equivalence of linear complementarity problems," Operations Research Letters, vol. 30, no. 4, pp. 211222, Aug. 2002. 


\title{
On the Equivalence of Linear Complementarity Problems
}

\author{
B. De Schutter ${ }^{\dagger *}$ W.P.M.H. Heemels ${ }^{\ddagger}$, A. Bemporad ${ }^{\S}$ \\ ${ }^{\dagger}$ Control Systems Engineering, Fac. ITS, Delft University of Technology, \\ P.O. Box 5031, 2600 GA Delft, The Netherlands, \\ tel: +31-15-27851 13, fax: +31-15-27866 79, b.deschutter@its.tudelft.nl \\ ${ }^{\ddagger}$ Dept. of Electrical Eng., Eindhoven Univ. of Technology, P.O. Box 513, 5600 MB Eindhoven, \\ The Netherlands, tel: +31-40-247 35 87, fax: +31-40-243 45 82, m.heemels@tue.nl \\ $\S$ Dip. Ingegneria dell'Informazione, Università di Siena, Via Roma 56, 53100 Siena, Italy, \\ tel: +39-0577-234631, fax: +39-0577-234632, bemporad@di.unisi.it
}

\begin{abstract}
We show that the Extended Linear Complementarity Problem (ELCP) can be recast as a standard Linear Complementarity Problem (LCP) provided that the surplus variables or the feasible set of the ELCP are bounded. Since many extensions of the LCP are special cases of the ELCP, this implies that these extensions can be rewritten as an LCP as well. Our equivalence proof is constructive and leads to three possible numerical solution methods for a given ELCP: regular ELCP algorithms, mixed integer linear programming algorithms, and regular LCP algorithms.
\end{abstract}

Keywords: complementarity problems, nonlinear algorithms, integer programming, optimization, linear complementarity problem

\footnotetext{
${ }^{*}$ Corresponding author.
} 


\section{Introduction}

The Linear Complementarity Problem (LCP) is one of the fundamental problems in mathematical programming $[6,36]$. Several authors have introduced (both linear and nonlinear) extensions of the LCP $[5-7,14,16,20,21,23,33,36,37,42,47]$. The importance of the LCP and its generalizations is evidenced by a broad range of applications in the fields of engineering and economics such as quadratic programming problems,, determination of Nash equilibria, nonlinear obstacle problems, and problems involving market equilibria, invariant capital stock, optimal stopping, contact and structural mechanics, elastohydrodynamic lubrication, and traffic equilibria $[6,17,18]$.

In [9] we have introduced yet another extension of the LCP, which we have called the Extended Linear Complementarity Problem (ELCP). This problem arose from our research on discrete event systems (max-plus-linear systems, max-plus-algebraic applications, and minmax-plus systems $[10,13]$ ) and hybrid systems (traffic signal control, and first-order hybrid systems with saturation $[8,12])$. Furthermore, the ELCP can also be used in the analysis of a class of hybrid systems called complementary slackness systems or linear complementarity systems $[11,25,39,45]$.

Besides the applications of ELCP in the domain of discrete event and hybrid systems, its relevance is also demonstrated by the fact that the LCP and most of its "linear" extensions are actually special cases of the ELCP $[9,11]$. The objective of this paper is to show that the reverse statement holds as well: any ELCP can be recast as an LCP provided that the surplus variables of the inequalities of the ELCP are bounded over the feasible set (A sufficient condition for this is that the feasible set of the ELCP is bounded). This also implies directly that the "linear" extensions of the LCP that are a special case of the ELCP (such as, e.g., the Vertical LCP or the Horizontal LCP [6]) can be rewritten as an LCP (provided that their surplus variables are bounded over the feasible set). In addition, we show a semi-global equivalence between the ELCP and the LCP in the sense that for any arbitrarily large set the solutions of the ELCP that lie in that set can be retrieved via an LCP.

In the derivation of our result we obtain another equivalent problem, which will be called the mixed integer linear feasibility problem (MILFP). The equivalence proof is constructive as we present explicit expressions for the LCP and the MILFP that are equivalent to the original ELCP. As a consequence, our result leads to several ways to solve a given ELCP with bounded surplus variables or with solutions that lie in a bounded set: either as an ELCP using the algorithm we have derived in [9], as a mixed integer linear programming problem, or as a standard LCP using one of the many available LCP algorithms. The computation time of the ELCP algorithm of [9] increases rapidly as the number of (in)equalities and variables increases, which may often be prohibitive. However, in many applications we only need one solution of the ELCP. In these cases the alternative (mixed integer or LCP) algorithms offer an attractive, more efficient way to solve the ELCP.

\section{The LCP and the ELCP}

All vectors used in this paper are assumed to be column vectors. Furthermore, inequalities for vectors have to be interpreted componentwise. We use $I_{n}$ to denote the $n$ by $n$ identity matrix, and $0_{m \times n}$ to denote the $m$ by $n$ zero matrix. If the dimensions of the identity matrix or the zero matrix are omitted, they should be clear from the context. 


\subsection{Linear Complementarity Problem (LCP)}

One of the possible formulations of the LCP is the following [6]:

Given $M \in \mathbb{R}^{n \times n}$ and $q \in \mathbb{R}^{n}$, find vectors $w, z \in \mathbb{R}^{n}$ such that

$$
\begin{aligned}
& w=M z+q \\
& w, z \geq 0 \\
& w^{T} z=0 .
\end{aligned}
$$

Condition (3) is called the complementarity condition of the LCP. Sometimes we will rewrite (2)-(3) more compactly as $0 \leq w \perp z \geq 0$. For algorithms to solve the LCP we refer the interested reader to $[1,3,6,27-31,34,36,40,46]$ and the references therein.

\subsection{Extended Linear Complementarity Problem (ELCP)}

The ELCP is defined as follows [9]:

Given $A \in \mathbb{R}^{p \times n}, B \in \mathbb{R}^{q \times n}, c \in \mathbb{R}^{p}, d \in \mathbb{R}^{q}$, and $m$ index sets $\phi_{1}, \ldots, \phi_{m} \subseteq\{1, \ldots, p\}$, find $x \in \mathbb{R}^{n}$ such that

$$
\begin{aligned}
& A x \geq c \\
& B x=d \\
& \sum_{j=1}^{m} \prod_{i \in \phi_{j}}(A x-c)_{i}=0 .
\end{aligned}
$$

The feasible set of the ELCP (4)-(6) is defined by $\mathcal{F}=\left\{x \in \mathbb{R}^{n} \mid A x \geq c, B x=d\right\}$. The surplus variable $\operatorname{surp}(i, x)$ of the $i$ th inequality of $A x \geq c$ is defined as $\operatorname{surp}(i, x)=(A x-c)_{i}$.

Condition (6) represents the complementarity condition of the ELCP. One possible interpretation of this condition is the following. Since $A x \geq c$, all the terms in (6) are nonnegative. Hence, (6) is equivalent to $\prod_{i \in \phi_{j}}(A x-c)_{i}=0$ for $j=1, \ldots, m$. So we could say that each set $\phi_{j}$ corresponds to a group of inequalities in $A x \geq c$, and that in each group at least one inequality should hold with equality (i.e., the corresponding surplus variable is equal to 0 ).

Remark 2.1 We may without loss of generality assume that the ELCP is written as

$$
\begin{aligned}
& A x \geq c \\
& \sum_{i=1}^{m} \prod_{j \in \phi_{i}}(A x-c)_{j}=0,
\end{aligned}
$$

since we can, e.g., replace $B x=d$ by $B x \geq d$, and obtain equality conditions on these inequalities by adding the index sets $\phi_{m+1}=\{p+1\}, \ldots, \phi_{m+q}=\{p+q\}$. However, if we want to solve an ELCP using, e.g., the algorithm of [9], then the formulation (5)-(6) leads to a more efficient solution than the reformulation (7)-(8). 
Remark 2.2 The complementarity conditions of both the LCP and the ELCP consist of a sum of products. However, in contrast to the ELCP where the products may contain one, two or more factors, the products in complementarity condition of the LCP always contain exactly two factors. Moreover, any variable in the LCP is contained in precisely one index set $\phi_{j}$, while in the ELCP formulation it may be contained in any number of index sets.

In [9] we have developed an algorithm to find all solutions of an ELCP. This algorithm yields a description of the complete solution set of an ELCP by finite points, generators for extreme rays, and a basis for the linear subspace associated with the maximal affine subspace of the solution set of the ELCP.

\subsection{Extensions of the LCP}

Several authors have introduced linear and nonlinear extensions and generalizations of the LCP. Some examples of "linear" extensions of the LCP are: the Horizontal LCP [6], the Vertical LCP [6] (also known as the Generalized LCP of Cottle and Dantzig [5]), the Generalized LCP of De Moor and Vandenberghe [7], the Generalized Order LCP and the Extended Generalized Order LCP of Gowda and Sznajder [21], the Extended LCP of Mangasarian and Pang [20,33], the Generalized LCP of Ye [47], the mixed LCP [6], the Extended Horizontal LCP of Sznajder and Gowda [42], the Generalized LCP of Eaves [14], and the Linear Dynamic Complementarity Problem $[39,45]$. In $[9,11]$ we have shown that all these generalizations are special cases of the ELCP.

The underlying geometrical explanation for the fact that all the generalizations of the LCP mentioned above are particular cases of the ELCP is that they all have a solution set that consists of the union of faces of a polyhedron, and that the union of any arbitrary set of faces of an arbitrary polyhedron can be described by an ELCP [9]. More generally, if we define a "linear" generalization of the LCP as a problem consisting of an explicit or implicit system of linear (in)equalities in combination with a "general" complementarity condition (i.e., an ELCP-like complementarity condition that constrains the solutions of the problem to lie on the (relative) boundary of the feasible set), then the solution set of this "linear" generalization will consist of the union of faces of a polyhedron, which implies that such a "linear" generalization of the LCP is a special case of the ELCP.

\section{The link between the LCP and ELCP}

Lemma 3.1 The LCP is a special case of the ELCP.

Proof: If we set $x=\left[\begin{array}{c}w \\ z\end{array}\right], A=I_{2 n}, B=\left[\begin{array}{ll}I_{n} & -M\end{array}\right], c=0_{2 n \times 1}, d=q$ and $\phi_{j}=\{j, j+n\}$ for $j=1, \ldots, n$ in the formulation of the ELCP, we get an LCP.

Theorem 3.2 If the surplus variables of the inequalities of an ELCP are bounded (from above $^{1}$ ) over the feasible set of the ELCP, then the ELCP can be rewritten as an $L C P$.

\footnotetext{
${ }^{1}$ We only need boundedness from above since the surplus variables are always nonnegative due to the condition $A x \geq c$.
} 
Proof: Consider the ELCP (4)-(6). If there is an equality condition $B x=d$ present, then we remove it using the procedure given in Remark 2.1. So from now on we consider the formulation (7)-(8) of the ELCP.

The proof will be done in two steps. First, we will transform the ELCP into a mixed integer problem to get rid of the ELCP complementarity condition at the cost of introducing some additional binary variables. In the second step we will transform all variables (both binary and real-valued) into nonnegative real ones, which will lead to an LCP.

Define a diagonal matrix $D^{\text {upp }} \in \mathbb{R}^{p \times p}$ with $\left(D^{\text {upp }}\right)_{i i}=d_{i i}^{\text {upp }}$ an upper bound for $\operatorname{surp}(i, x)=$ $(A x-c)_{i}$ over the feasible set $\mathcal{F}$ of the ELCP. Now consider the following system of equations:

$$
\begin{array}{ll}
\delta \in\{0,1\}^{p}, x \in \mathbb{R}^{n} & \\
0 \leq(A x-c)_{i} \leq d_{i i}^{\mathrm{upp}} \delta_{i} & \text { for } i=1, \ldots, p, \\
\sum_{i \in \phi_{j}} \delta_{i} \leq \# \phi_{j}-1 & \text { for } j=1, \ldots, m,
\end{array}
$$

where $\# \phi_{j}$ denotes the number of elements of the set $\phi_{j}$. Problem (9)-(11) will be called the equivalent mixed integer linear feasibility problem (MILFP).

Now we show that the MILFP is equivalent to the ELCP (7)-(8) in the sense that a vector $x$ is a solution of the ELCP (7)-(8) if and only if there exists a vector $\delta$ such that $(x, \delta)$ is a solution of (9)-(11). Equation (7) is implied by (10). Note that (9) and (11) imply that for each $j$ at least one of the $\delta_{i}$ 's with $i \in \phi_{j}$ is equal to 0 . If $\delta_{i^{\prime}}=0$, then it follows from (10) that $(A x-c)_{i^{\prime}}=0$. This implies that in each index set $\phi_{j}$ there is at least one index for which the corresponding surplus variable equals 0 . Hence, the complementarity condition (8) is also implied by (9)-(11). So (9)-(11) imply (7)-(8), and it is easy to verify that the reverse statement also holds. As a consequence, the MILFP is equivalent to the ELCP.

Define a matrix $S \in \mathbb{R}^{m \times p}$ with $s_{j i}=1$ if $i \in \phi_{j}$ and $s_{j i}=0$ otherwise, and a vector $t \in \mathbb{R}^{m}$ with $t_{j}=\# \phi_{j}-1$. The MILFP can now be rewritten compactly as

$$
\begin{aligned}
& \delta \in\{0,1\}^{p}, x \in \mathbb{R}^{n} \\
& 0 \leq A x-c \leq D^{\text {upp }} \delta \\
& S \delta \leq t .
\end{aligned}
$$

Now we will transform this problem into an LCP. This will be done in three steps.

1. First we transform condition (12) into the LCP framework. All the variables of an LCP should be real-valued, but the vector $\delta$ in the MILFP is a binary vector. However, the condition $\delta_{i} \in\{0,1\}$ is equivalent to $0 \leq \delta_{i} \perp 1-\delta_{i} \geq 0$. So if we introduce a vector $v_{\delta} \in \mathbb{R}^{p}$ of auxiliary variables, then the condition $\delta \in\{0,1\}^{p}$ is equivalent to

$$
\delta, v_{\delta} \in \mathbb{R}^{p}, \quad v_{\delta}=\mathbf{1}_{p}-\delta, \quad 0 \leq \delta \perp v_{\delta} \geq 0,
$$

where $\mathbf{1}_{p}$ is a $p$-component column vector consisting of all 1's.

2. The inequality $0 \leq A x-c$ can be adapted to the LCP framework by introducing an auxiliary vector $v_{A} \in \mathbb{R}^{p}$ with $v_{A}=A x-c \geq 0$. To obtain a complementarity condition for $v_{A}$ we introduce $w_{A} \in \mathbb{R}^{p}$ such that $0 \leq v_{A} \perp w_{A} \geq 0$ (Note that we can always take $w_{A}=0$ to get this condition satisfied). Hence, $0 \leq A x-c$ can be rewritten as

$$
v_{A}=A x-c, \quad 0 \leq v_{A} \perp w_{A} \geq 0
$$


with $v_{A}, w_{A} \in \mathbb{R}^{p}$. The inequalities $A x-c \leq D^{\text {upp }} \delta$ and $S \delta \leq t$ can be dealt with in a similar way.

3. All variables in an LCP are nonnegative whereas this condition is not present in the MILFP or the ELCP. Therefore, we split $x$ in its positive part $x^{+}=\max (x, 0)$ and its negative part $x^{-}=\max (-x, 0)$. So $x=x^{+}-x^{-}$and $0 \leq x^{+} \perp x^{-} \geq 0$. To obtain independent complementarity conditions for $x^{+}$and $x^{-}$we introduce additional auxiliary vectors $v^{+}, v^{-} \in \mathbb{R}^{n}$ with $v^{+}=x^{+}$and $v^{-}=x^{-}$such that $0 \leq v^{-} \perp x^{+} \geq 0$ and $0 \leq v^{+} \perp x^{-} \geq 0$.

Combining the three steps results in the following equivalent LCP:

$$
\begin{aligned}
& \underbrace{\left[\begin{array}{c}
v_{\delta} \\
v^{-} \\
v^{+} \\
v_{A} \\
v_{D^{\text {upp }}} \\
v_{S}
\end{array}\right]}_{w}=\underbrace{\left[\begin{array}{rrrrrr}
-I_{p} & 0 & 0 & 0 & 0 & 0 \\
0 & 0 & I_{n} & 0 & 0 & 0 \\
0 & I_{n} & 0 & 0 & 0 & 0 \\
0 & A & -A & 0 & 0 & 0 \\
D^{\mathrm{upp}} & -A & A & 0 & 0 & 0 \\
-S & 0 & 0 & 0 & 0 & 0
\end{array}\right]}_{M} \underbrace{\left[\begin{array}{c}
\delta \\
x^{+} \\
x^{-} \\
w_{A} \\
w_{D^{\mathrm{upp}}} \\
w_{S}
\end{array}\right]}_{z}+\underbrace{\left[\begin{array}{c}
\mathbf{1}_{p} \\
0 \\
0 \\
-c \\
c \\
t
\end{array}\right]}_{q} \\
& 0 \leq w \perp z \geq 0
\end{aligned}
$$

with $w, z \in \mathbb{R}^{3 p+2 n+m}$. The solution of the original ELCP can be extracted from the solution of the LCP (15)-(16) by setting $x=x^{+}-x^{-}$.

The introduction of the MILFP in this proof is inspired by the paper [2], in which a class of hybrid systems is discussed consisting of mixed logical dynamic systems, which can be shown to be equivalent to systems with an ELCP-based model description [24].

The removal of the equality condition $B x=d$ in the proof above is only done to simplify the proof. If we want to use this proof to solve an ELCP by reformulating it as an MILFP, it is numerically better to keep the equalities. In that case the full ELCP (4)-(6) would result in the MILFP (9)-(11) but with the additional constraint $B x=d$.

If we are only interested in obtaining one solution of an ELCP, we can transform the corresponding MILFP into a mixed integer linear programming problem by adding a dummy linear objective function. This problem can then be solved using, e.g., a branch-and-bound method $[19,43]$ or a branch-and-cut method [4].

The matrix $M$ of the LCP (15)-(16) is not positive definite. Moreover, this LCP is in general not strictly feasible (i.e., in general the set $\left\{z \in \mathbb{R}^{n} \mid M z+q>0, z>0\right\}$ is empty). This may prevent us from using fast and efficient LCP methods such as, e.g., Lemke's method or strictly feasible interior point methods to solve the LCP (15)-(16). So then we may have to use alternative approaches such as the mixed integer programming approach of $[38,41]$ or a reformulation as a quadratic programming problem [6] (cf. the example in the appendix).

A sufficient condition for the surplus variables of the inequalities of the ELCP to be bounded is that the feasible set $\mathcal{F}$ of the ELCP is bounded. However, note that boundedness of the feasible set is not a necessary condition for boundedness of the surplus variables over the feasible set. Consider, e.g., the ELCP $x \in \mathbb{R}^{2}, 1 \leq x_{1} \leq 2,\left(x_{1}-1\right)\left(2-x_{1}\right)=0$, which has an unbounded feasible set $\mathcal{F}=\left\{x \in \mathbb{R}^{2} \mid x_{1}=1\right.$ or $\left.x_{1}=2\right\}$, but bounded surplus variables $\operatorname{surp}(1, x), \operatorname{surp}(2, x) \in[0,1]$ over the feasible set $\mathcal{F}$.

Upper bounds for the surplus variables over the feasible set can be determined as follows: 
- The upper bounds $d_{j j}^{\text {upp }}$ can be computed efficiently using a linear programming problem:

$$
d_{i i}^{\text {upp }}=\max _{A x-c \geq 0}(A x-c)_{i} \quad \text { for } i=1, \ldots, p .
$$

If any of the $p$ linear programming problems yields an unbounded objective function $\left(d_{i i}^{\text {upp }} \rightarrow \infty\right)$, then the ELCP does not have a bounded feasible set and then the condition of Theorem 3.2 does not hold.

- If we know upper bounds $x^{\text {upp }}$ and lower bounds $x^{\text {low }}$ for the components of $x$, e.g., as a consequence of physical or other constraints, then we can even more efficiently compute upper bounds as

$$
d_{i i}^{\text {upp }}=\left(A^{+} x^{\text {upp }}-A^{-} x^{\text {low }}-c\right)_{i} \quad \text { for } i=1, \ldots, p,
$$

with $A^{+}$and $A^{-}$the positive and the negative part of $A$ (i.e., $A^{+}, A^{-} \in \mathbb{R}^{p \times n}$ with $\left(A^{+}\right)_{i j}=\max \left(a_{i j}, 0\right)$ and $\left(A^{-}\right)_{i j}=\max \left(-a_{i j}, 0\right)$ for all $\left.i, j\right)$. So $A=A^{+}-A^{-}$.

Even if we have an ELCP for which the surplus variables are not bounded over the feasible set, then in practical applications it often occurs that we are only interested in solutions that lie in a given (bounded) region. In that case we can use the following theorem which can be proved in a similar way as Theorem 3.2.

Theorem 3.3 Consider the ELCP (4)-(6) and let $X$ be an arbitrary bounded subset of $\mathbb{R}^{n}$. Then every solution $x \in X$ of the ELCP can be obtained as a solution of an equivalent MILFP or LCP (where the upper bounds $d_{i i}^{\text {upp }}$ are now defined as upper bounds of the surplus variables over the set $X$ instead of over the feasible set $\mathcal{F}$ of the $E L C P)$.

Note that Theorem 3.3 provides an equivalence between the ELCP and the LCP (or MILFP) in a semi-global sense: for any arbitrarily large set $X$, we can provide the solutions (or say that there are none) of an ELCP via the equivalent MILFP or LCP.

As mentioned before, several extensions of the LCP (which we have called "linear" generalizations of the LCP in Section 2.3) are special cases of the ELCP. So if the surplus variables or the feasible set of such a "linear" generalization of the LCP are bounded, then it follows from Theorem 3.2 that it can be rewritten as an LCP.

Several other authors have also studied the link between generalizations of the LCP and the standard LCP:

- Some equivalence results such as, e.g., between the mixed LCP and the standard LCP [6] require non-singularity conditions for certain matrices. Note that such conditions are not present in our equivalence result.

- In $[6,22]$ it has been shown that (variants of) the Horizontal LCP can be recast as an LCP. In addition, in [15] it was shown that an Horizontal LCP can be written as a system of piecewise linear equations (with real-valued variables), and vice versa. In [26] it is shown that under certain conditions (a special case of) the Generalized LCP of Ye [47] is equivalent to an LCP. Moreover, in [44] an algorithm is presented to reduce a Horizontal LCP to an LCP whenever it is possible to do so. However, in all these extensions of the LCP a "conventional" complementarity condition of the form $0 \leq w \perp z \geq 0$ is used instead of the more complex ELCP complementarity condition. 
- In $[32,35]$ it has been shown that the Vertical LCP can be reformulated as an LCP.

However, note that our result is more general since the ELCP can be considered as a unifying framework for all "linear" extensions of the LCP.

Moreover, several authors have already considered the link between a standard LCP and a mixed integer programming problem $[38,41]$. However, to the authors' best knowledge, the link between the ELCP and a mixed integer programming problem has not yet been considered previously.

\section{Conclusion}

We have shown that an ELCP with bounded surplus variables over the feasible set (or with a bounded feasible set) can be rewritten as an LCP. This also implies that many linear extensions of the LCP can be recast as an LCP under the same assumption. In addition, we have also shown an equivalence between the ELCP and the LCP in a semi-global sense: for any arbitrarily large set we can provide the solutions (or say that there are none) of an ELCP via an equivalent LCP. In our constructive equivalence proof we have introduced a mixed integer linear feasibility problem that is also equivalent to the ELCP. As a consequence, we now have three different ways to solve an ELCP: using the ELCP algorithm of [9], using a mixed integer (linear programming) algorithm, or using one of the many LCP algorithms. The main advantage of the latter two approaches is that they can be used for applications in which we only need one solution of the ELCP since in that case they will be much more efficient than the ELCP algorithm of [9], which computes all solutions and, as a consequence, requires much more computation time.

An important topic for further research is a thorough evaluation and comparison of the performance of several different mixed integer and LCP algorithms for the special cases of the ELCP that arise in specific applications involving discrete event systems and hybrid systems. As our equivalence proof was constructive, we have obtained explicit LCP and MILFP expressions for a given ELCP. The resulting LCP or MILFP reformulation is not necessarily the most efficient one (i.e., with a minimal number of variables or equations). This causes no problems if we only want to prove the conceptual equivalence, which was the intention of this paper. However, in case we aim at using the equivalent LCP or MILFP to solve the original ELCP in a computationally very efficient way, then it might be useful to look for techniques to reduce the LCP or MILFP by removing redundant variables or inequalities before actually solving the LCP or MILFP. This will also be a topic for future research.

\section{Acknowledgment}

Research partially funded by the Dutch Technology Foundation STW project "Model predictive control for hybrid systems" (DMR.5675).

\section{References}

[1] Z.Z. Bai. On the convergence of the multisplitting methods for the linear complementarity problem. SIAM Journal on Matrix Analysis and Applications, 21(1):67-78, 1999.

[2] A. Bemporad and M. Morari. Control of systems integrating logic, dynamics, and constraints. Automatica, 35(3):407-427, March 1999. 
[3] C. Chen and O.L. Mangasarian. Smoothing methods for convex inequalities and linear complementarity problems. Mathematical Programming, 71(1):51-69, November 1995.

[4] C. Cordier, H. Marchand, R. Laundy, and L.A. Wolsey. bc-opt: A branch-and-cut code for mixed integer programs. Mathematical Programming, Series A, 86(2):335-353, 1999.

[5] R.W. Cottle and G.B. Dantzig. A generalization of the linear complementarity problem. Journal of Combinatorial Theory, 8(1):79-90, January 1970.

[6] R.W. Cottle, J.S. Pang, and R.E. Stone. The Linear Complementarity Problem. Academic Press, Boston, Massachusetts, 1992.

[7] B. De Moor, L. Vandenberghe, and J. Vandewalle. The generalized linear complementarity problem and an algorithm to find all its solutions. Mathematical Programming, 57:415-426, 1992.

[8] B. De Schutter. Optimal control of a class of linear hybrid systems with saturation. SIAM Journal on Control and Optimization, 39(3):835-851, 2000.

[9] B. De Schutter and B. De Moor. The extended linear complementarity problem. Mathematical Programming, 71(3):289-325, December 1995.

[10] B. De Schutter and B. De Moor. Minimal realization in the max algebra is an extended linear complementarity problem. Systems $\& 3$ Control Letters, 25(2):103-111, May 1995.

[11] B. De Schutter and B. De Moor. The Linear Dynamic Complementarity Problem is a special case of the Extended Linear Complementarity Problem. Systems \& Control Letters, 34(1-2):63-75, May 1998.

[12] B. De Schutter and B. De Moor. Optimal traffic light control for a single intersection. European Journal of Control, 4(3):260-276, 1998.

[13] B. De Schutter and T. van den Boom. Model predictive control for max-min-plus systems. In R. Boel and G. Stremersch, editors, Discrete Event Systems: Analysis and Control (Proceedings of the 5th International Workshop on Discrete Event Systems (WODES2000), Ghent, Belgium, August 2000), volume 569 of The Kluwer International Series in Engineering and Computer Science, pages 201-208. Kluwer Academic Publishers, Boston, Massachusetts, 2000.

[14] B.C. Eaves. The linear complementarity problem. Management Science, 17(9):612-634, May 1971.

[15] B.C. Eaves and C.E. Lemke. Equivalence of LCP and PLS. Mathematics of Operations Research, 6(4):475-484, November 1981.

[16] M.C. Ferris and C. Kanzow. Complementarity and related problems. In P.M. Pardalos and M.G.C. Resende, editors, Handbook of Applied Optimization, chapter 13, pages 514530. Oxford University Press, Oxford, UK, 2002.

[17] M.C. Ferris and J.S. Pang, editors. Complementarity and Variational Problems: State of the Art. Philadelphia, Pennsylvania: SIAM, 1997. (Proceedings of the International Conference on Complementarity Problems, Baltimore, Maryland, November 1995). 
[18] M.C. Ferris and J.S. Pang. Engineering and economic applications of complementarity problems. SIAM Review, 39(4):669-713, December 1997.

[19] R. Fletcher and S. Leyffer. Numerical experience with lower bounds for MIQP branchand-bound. SIAM Journal on Optimization, 8(2):604-616, May 1998.

[20] M.S. Gowda. On the extended linear complementarity problem. Mathematical Programming, 72:33-50, 1996.

[21] M.S. Gowda and R. Sznajder. The generalized order linear complementarity problem. SIAM Journal on Matrix Analysis and Applications, 15(3):779-795, July 1994.

[22] O. Güler. Generalized linear complementarity problems. Mathematics of Operations Research, 20(2):441-448, May 1995.

[23] P.T. Harker and J.S. Pang. Finite-dimensional variational inequality and nonlinear complementarity problems: A survey of theory, algorithms and applications. Mathematical Programming, Series B, 48(2):161-220, September 1990.

[24] W.P.M.H. Heemels, B. De Schutter, and A. Bemporad. Equivalence of hybrid dynamical models. Automatica, 37(7):1085-1091, July 2001.

[25] W.P.M.H. Heemels, J.M. Schumacher, and S. Weiland. Linear complementarity systems. SIAM Journal on Applied Mathematics, 60(4):1234-1269, 2000.

[26] J.J. Júdice and L.N. Vicente. On the solution and complexity of a generalized linear complementarity problem. Journal of Global Optimization, 4(4):415-424, 1994.

[27] J.A. Kaliski and Y. Ye. An extension of the potential reduction algorithm for linear complementarity problems with some priority goals. Linear Algebra and Its Applications, 193:35-50, 1993.

[28] C. Kanzow. Some noninterior continuation methods for linear complementarity problems. SIAM Journal on Matrix Analysis and Applications, 17(4):851-868, October 1996.

[29] M. Kočvara and J. Zowe. An iterative two-step algorithm for linear complementarity problems. Numerische Mathematik, 68(1):95-106, June 1994.

[30] H. Kremers and D. Talman. A new pivoting algorithm for the linear complementarity problem allowing for an arbitrary starting point. Mathematical Programming, 63(2):235252, January 1994.

[31] D.M.W. Leenaerts and W.M.G. van Bokhoven. Piecewise Linear Modeling and Analysis. Kluwer Academic Publishers, Boston, Massachusetts, 1998.

[32] C.E. Lemke. Recent results on complementarity problems. In J.B. Rosen, O.L. Mangasarian, and K. Ritter, editors, Nonlinear Programming (Proceedings of a symposium conducted by the Mathematics Research Center, University of Wisconsin, Madison, Wisconsin, May 1970), pages 349-384, New York, 1970. Academic Press.

[33] O.L. Mangasarian and J.S. Pang. The extended linear complementarity problem. SIAM Journal on Matrix Analysis and Applications, 16(2):359-368, January 1995. 
[34] S. Mehrotra and R.A. Stubbs. Predictor-corrector methods for a class of linear complementarity problems. SIAM Journal on Optimization, 4(2):441-453, May 1994.

[35] S.R. Mohan, S.K. Neogy, and R. Sridhar. The generalized linear complementarity problem revisited. Mathematical Programming, 74:197-218, 1996.

[36] K.G. Murty. Linear Complementarity, Linear and Nonlinear Programming. Helderman Verlag, Berlin, Germany, 1988.

[37] J.S. Pang and S.A. Gabriel. NE/SQP: A robust algorithm for the nonlinear complementarity problem. Mathematical Programming, 60(3):295-337, July 1993.

[38] P.M. Pardalos. Linear complementarity problems solvable by integer programming. $O p$ timization, 19(4):467-474, 1988.

[39] J.M. Schumacher. Some modeling aspects of unilaterally constrained dynamics. In Proceedings of the ESA International Workshop on Advanced Mathematical Methods in the Dynamics of Flexible Bodies, ESA-ESTEC, Noordwijk, The Netherlands, June 1996.

[40] R. Sheng and F.A. Potra. A quadratically convergent infeasible-interior-point algorithm for LCP with polynomial complexity. SIAM Journal on Optimization, 7(2):304-317, May 1997.

[41] H.D. Sherali, R.S. Krishnamurthy, and F.A. Al-Khayyal. Enhanced intersection cuttingplane approach for linear complementarity problems. Journal of Optimization Theory and Applications, 90(1):183-201, July 1996.

[42] R. Sznajder and M.S. Gowda. Generalizations of $P_{0^{-}}$and $P$-properties; extended vertical and horizontal linear complementarity problems. Linear Algebra and Its Applications, 223/224:695-715, July 1995.

[43] H.A. Taha. Operations Research: An Introduction. Macmillan Publishing Company, New York, 4th edition, 1987.

[44] R.H. Tütüncü and M.J. Todd. Reducing horizontal linear complementarity problems. Linear Algebra and Its Applications, 223/224:717-729, July 1995.

[45] A.J. van der Schaft and J.M. Schumacher. Hybrid systems modelling and complementarity problems. In Proceedings of the European Control Conference (ECC'97), Brussels, Belgium, paper 868, July 1997.

[46] S.J. Wright. An infeasible-interior-point algorithm for linear complementarity problems. Mathematical Programming, 67(1):29-51, October 1994.

[47] Y. Ye. A fully polynomial-time approximation algorithm for computing a stationary point of the general linear complementarity problem. Mathematics of Operations Research, 18(2):334-345, May 1993. 


\section{Appendix: Example}

Consider the following ELCP:

Find $x \in \mathbb{R}^{3}$ such that

$$
\begin{aligned}
x_{1} & \geq 1 \\
-x_{1} & \geq-2 \\
x_{2} & \geq 1 \\
-x_{2}+4 x_{3} & \geq-1 \\
-x_{1}-2 x_{2}-2 x_{3} & \geq-13
\end{aligned}
$$

subject to

$$
\operatorname{surp}(1, x) \operatorname{surp}(2, x) \operatorname{surp}(5, x)+\operatorname{surp}(2, x) \operatorname{surp}(3, x)+\operatorname{surp}(4, x)=0 .
$$

The feasible set $\mathcal{F}$ of this ELCP is presented in Figure 1 and corresponds to the polyhedron with the following vertices:

$$
e_{1}=\left[\begin{array}{l}
1 \\
1 \\
0
\end{array}\right], e_{2}=\left[\begin{array}{l}
2 \\
1 \\
0
\end{array}\right], e_{3}=\left[\begin{array}{l}
1 \\
1 \\
5
\end{array}\right], e_{4}=\left[\begin{array}{l}
1 \\
5 \\
1
\end{array}\right], e_{5}=\left[\begin{array}{c}
2 \\
1 \\
4.5
\end{array}\right], e_{6}=\left[\begin{array}{c}
2 \\
4.6 \\
0.9
\end{array}\right]
$$

The faces of the polyhedron $\mathcal{F}$ correspond to the boundary hyperplanes of the inequalities (19)-(23). The solutions of the ELCP are then the points of the polyhedron $\mathcal{F}$ that satisfy the complementarity condition (24). It is easy to verify that (24) will be satisfied if

- the 1st, 3rd and 4th surplus variable are all 0 (which corresponds to the intersection of the boundary hyperplanes of (19), (21) and (22), i.e., the point $e_{1}$ ),

- the 2 nd and the 4 th surplus variable are all 0 (which corresponds to the intersection of the boundary hyperplanes of $(20)$ and (22), i.e., the line $\left.e_{2} e_{6}\right)$.

The other combinations (1-2-4, 2-3-4, 5-2-4, and 5-3-4) are either implied by the combinations given above, or do not lead to a feasible solution since the corresponding hyperplanes intersect outside the feasible set. So the solution set of the ELCP is given by

$$
\mathcal{S}=\left\{e_{1}\right\} \cup e_{2} e_{6}=\left\{\left[\begin{array}{l}
1 \\
1 \\
0
\end{array}\right]\right\} \cup\left\{\lambda\left[\begin{array}{l}
2 \\
1 \\
0
\end{array}\right]+(1-\lambda)\left[\begin{array}{c}
2 \\
4.6 \\
0.9
\end{array}\right] \mid \lambda \in[0,1]\right\} .
$$

In Figure 1 the solution set $\mathcal{S}$ of the ELCP (19)-(24) is indicated by the thick dot and the thick line.

The feasible set of the ELCP is bounded so that we can apply Theorem 3.2. For the ELCP (19)-(24) we have

$$
A=\left[\begin{array}{rrr}
1 & 0 & 0 \\
-1 & 0 & 0 \\
0 & 1 & 0 \\
0 & -1 & 4 \\
-1 & -2 & -2
\end{array}\right], c=\left[\begin{array}{r}
1 \\
-2 \\
1 \\
-1 \\
-13
\end{array}\right], \phi_{1}=\{1,2,5\}, \phi_{2}=\{2,3\} \text { and } \phi_{3}=\{4\}
$$




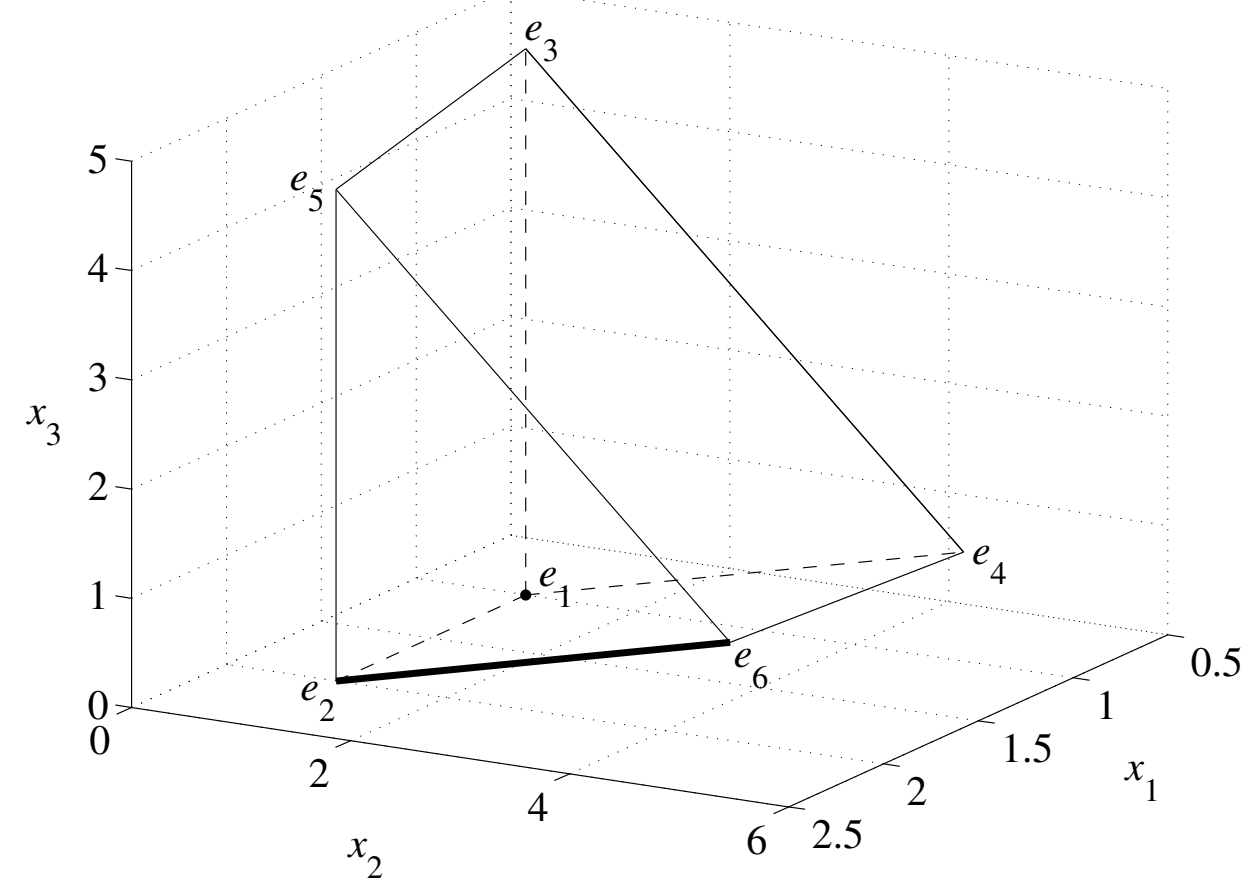

Figure 1: The feasible set $\mathcal{F}$ with vertices $e_{1}, e_{2}, \ldots e_{6}$ of the ELCP (19)-(24) and the solution set $\left\{e_{1}\right\} \cup e_{2} e_{6}$ of the ELCP (indicated by the thick dot and the thick line).

Furthermore, (17) yields the following upper bounds $d_{j j}^{\text {upp }}$ for $(A x-c)_{j}$ over the feasible set of the $\mathrm{ELCP}^{2}:\left\{d_{j j}^{\mathrm{upp}}\right\}_{j=1, \ldots, 5}=1,1,4,20,10$. So we can rewrite the ELCP as the MILFP (12)-(14) with

$$
D^{\text {upp }}=\left[\begin{array}{ccccc}
1 & 0 & 0 & 0 & 0 \\
0 & 1 & 0 & 0 & 0 \\
0 & 0 & 4 & 0 & 0 \\
0 & 0 & 0 & 20 & 0 \\
0 & 0 & 0 & 0 & 10
\end{array}\right], S=\left[\begin{array}{ccccc}
1 & 1 & 0 & 0 & 1 \\
0 & 1 & 1 & 0 & 0 \\
0 & 0 & 0 & 1 & 0
\end{array}\right], t=\left[\begin{array}{l}
2 \\
1 \\
0
\end{array}\right],
$$

and with $x \in \mathbb{R}^{3}, \delta \in\{0,1\}^{5}$. If introduce the dummy objective function $x_{1}+x_{2}+x_{3}+$ $\delta_{1}+\delta_{2}+\delta_{3}+\delta_{4}+\delta_{5}$, we get a mixed integer linear programming problem. If we use a branch-and-bound algorithm to solve this problem, we obtain the following solution:

$$
x_{\mathrm{MILFP}}=\left[\begin{array}{l}
1 \\
1 \\
0
\end{array}\right], \quad \delta_{\mathrm{MILFP}}=\left[\begin{array}{l}
0 \\
1 \\
0 \\
0 \\
1
\end{array}\right] .
$$

Note that $x_{\mathrm{MILFP}}=e_{1}$ belongs to $\mathcal{S}$.

\footnotetext{
${ }^{2}$ Using formula (18) for the upper bound $x^{\text {upp }}=\left[\begin{array}{lll}2 & 5 & 5\end{array}\right]^{T}$ and the lower bound $x^{\text {low }}=\left[\begin{array}{lll}1 & 1 & 0\end{array}\right]^{T}$ yields the same result.
} 
Finally, we can rewrite the MILFP as an LCP (15)-(16) with $w, z \in \mathbb{R}^{24}$. We have $M=\left[\begin{array}{ll}M_{1} & 0_{24 \times 13}\end{array}\right]$ with

$$
M_{1}=\left[\begin{array}{rrrrrrrrrrr}
-1 & 0 & 0 & 0 & 0 & 0 & 0 & 0 & 0 & 0 & 0 \\
0 & -1 & 0 & 0 & 0 & 0 & 0 & 0 & 0 & 0 & 0 \\
0 & 0 & -1 & 0 & 0 & 0 & 0 & 0 & 0 & 0 & 0 \\
0 & 0 & 0 & -1 & 0 & 0 & 0 & 0 & 0 & 0 & 0 \\
0 & 0 & 0 & 0 & -1 & 0 & 0 & 0 & 0 & 0 & 0 \\
0 & 0 & 0 & 0 & 0 & 0 & 0 & 0 & 1 & 0 & 0 \\
0 & 0 & 0 & 0 & 0 & 0 & 0 & 0 & 0 & 1 & 0 \\
0 & 0 & 0 & 0 & 0 & 0 & 0 & 0 & 0 & 0 & 1 \\
0 & 0 & 0 & 0 & 0 & 1 & 0 & 0 & 0 & 0 & 0 \\
0 & 0 & 0 & 0 & 0 & 0 & 1 & 0 & 0 & 0 & 0 \\
0 & 0 & 0 & 0 & 0 & 0 & 0 & 1 & 0 & 0 & 0 \\
0 & 0 & 0 & 0 & 0 & 1 & 0 & 0 & -1 & 0 & 0 \\
0 & 0 & 0 & 0 & 0 & -1 & 0 & 0 & 1 & 0 & 0 \\
0 & 0 & 0 & 0 & 0 & 0 & 1 & 0 & 0 & -1 & 0 \\
0 & 0 & 0 & 0 & 0 & 0 & -1 & 4 & 0 & 1 & -4 \\
0 & 0 & 0 & 0 & 0 & -1 & -2 & -2 & 1 & 2 & 2 \\
1 & 0 & 0 & 0 & 0 & -1 & 0 & 0 & 1 & 0 & 0 \\
0 & 1 & 0 & 0 & 0 & 1 & 0 & 0 & -1 & 0 & 0 \\
0 & 0 & 4 & 0 & 0 & 0 & -1 & 0 & 0 & 1 & 0 \\
0 & 0 & 0 & 20 & 0 & 0 & 1 & -4 & 0 & -1 & 4 \\
0 & 0 & 0 & 0 & 10 & 1 & 2 & 2 & -1 & -2 & -2 \\
-1 & -1 & 0 & 0 & -1 & 0 & 0 & 0 & 0 & 0 & 0 \\
0 & -1 & -1 & 0 & 0 & 0 & 0 & 0 & 0 & 0 & 0 \\
0 & 0 & 0 & -1 & 0 & 0 & 0 & 0 & 0 & 0 & 0
\end{array}\right] \quad \text { and } \quad q=\quad\left[\begin{array}{r}
1 \\
1 \\
1 \\
1 \\
1 \\
0 \\
0 \\
0 \\
0 \\
0 \\
0 \\
-1 \\
2 \\
-1 \\
1 \\
13 \\
1 \\
-2 \\
1 \\
-1 \\
-13 \\
2 \\
1 \\
0
\end{array}\right]
$$

If we solve this LCP using a quadratic programming approach ${ }^{3}[6]$, we get

$$
\delta_{\mathrm{LCP}}=\left[\begin{array}{l}
0 \\
1 \\
0 \\
0 \\
1
\end{array}\right], x_{\mathrm{LCP}}^{+}=\left[\begin{array}{l}
1 \\
1 \\
0
\end{array}\right], x_{\mathrm{LCP}}^{-}=\left[\begin{array}{l}
0 \\
0 \\
0
\end{array}\right] \text {, }
$$

and thus

$$
x_{\mathrm{LCP}}=x_{\mathrm{LCP}}^{+}-x_{\mathrm{LCP}}^{-}=\left[\begin{array}{l}
1 \\
1 \\
0
\end{array}\right],
$$

which gives the same solution as the branch-and-bound algorithm for the MILFP. If we use the ELCP algorithm of [9] to solve the LCP, we obtain - after extraction of $x_{\mathrm{LCP}}$ - the complete solution set $\mathcal{S}$ of the original ELCP (19)-(24).

\footnotetext{
${ }^{3}$ Note that, e.g., Lemke's method is not guaranteed to give a solution of this LCP since the matrix $M$ is indefinite, and since there exist degenerate solutions of the LCP (i.e., solutions with $w_{i}=z_{i}=0$ for some indices $i$ ) (see [6] for more information). If we consider the quadratic programming problem (QP) $\min _{z \in \mathbb{R}^{24}}\left\{z^{T}(M z+q)=0, z \geq 0, M z+q \geq 0\right\}$, then it is easy to verify that the optimal QP solution $z_{\mathrm{QP}}$ yields a solution of the LCP if the value of the objective function in $z_{\mathrm{QP}}$ is equal to 0 .
} 